\title{
Mitochondrial transcription factors TFA, TFB1 and TFB2: A search for DNA variants/haplotypes and the risk of cardiac hypertrophy
}

\author{
Cristina Alonso-Montes ${ }^{\mathrm{a}}$, Mónica G. Castro ${ }^{\mathrm{a}}$, Julián R. Reguero ${ }^{\mathrm{b}}$, Andreas Perrot $^{\mathrm{c}}$, Cemil Özcelik ${ }^{\mathrm{c}}$, \\ Christian Geier ${ }^{\mathrm{c}}$, Maximilian G. Posch ${ }^{\mathrm{c}}$, César Morís ${ }^{\mathrm{b}}$, Victoria Alvarez ${ }^{\mathrm{a}}$, Marta Ruiz-Ortega ${ }^{\mathrm{d}, \mathrm{e}}$ and \\ Eliecer Coto ${ }^{\mathrm{a}, \mathrm{e}, \mathrm{f}, *}$ \\ ${ }^{a}$ Genética Molecular, Hospital Central Asturias, Oviedo, Spain \\ ${ }^{\mathrm{b}}$ Cardiología-Fundación Asturcor, Hospital Central Asturias, Oviedo, Spain \\ ${ }^{\mathrm{c}}$ Charité - Universitätsmedizin Berlin/Kardiologie am Campus Buch \& Virchow-Klinikum, Max-Delbrück-Centrum \\ für Molekulare Medizin, Berlin, Germany \\ ${ }^{\mathrm{d}}$ Cellular Biology in Renal Diseases Laboratory, Fundación Jiménez Díaz and Universidad Autónoma de Madrid, \\ Madrid, Spain \\ ${ }^{\mathrm{e}}$ Red de Investigación Renal-REDINREN, Spain \\ ${ }^{\mathrm{f}}$ Instituto Reina Sofía de Investigación Nefrológica, Spain
}

\begin{abstract}
Mitochondrial transcription factors mtTFA, mtTFB1 and mtTFB2 are required for the replication of mitochondrial DNA (mtDNA), regulating the number of mtDNA copies. Mice with a mtTFA deletion showed a reduced number of mtDNA copies, a reduction in respiratory chain activity, and a characteristic dilated cardiomyopathy. DNA variants in these genes could be involved in the risk for cardiac hypertrophy (HCM).

We determined the variation in the TFAM, TFB1M, and TFB2M genes (using SSCA, DHPLC, and direct sequencing) in a total of 200 HCM-patients from Spain and Germany, and in 250 healthy controls. We found several common polymorphisms that defined haplotype blocks in these genes, with frequencies that did not differ between patients and controls. We also found four novel variants in patients which were absent in the controls: $-91 \mathrm{C}>\mathrm{A}\left(5^{\prime}\right.$-UTR) and Ala105 > Thr in TFAM, and Thr211 > Ala and Arg256 > Lys in TFB1M. The three missense changes were in highly conserved amino acids, and could be involved in HCM-risk.

In conclusion, common variants in the mitochondrial transcription factors were not associated with the risk for HCM. However, rare DNA variants (putative mutations) could be involved in the pathogenesis of HCM in a reduced number of cases.
\end{abstract}

Keywords: Cardiac hypertrophy, mitochondria DNA, transcription factors, mutations

\section{Introduction}

Mitochondria are the cellular organelles that generate energy as adenosine triphosphate (ATP). Mitochon-

\footnotetext{
*Corresponding author: Eliecer Coto, Genética Molecular, HUCA-Maternidad, 33006-Oviedo, Spain. E-mail: eliecer.coto@ sespa.princast.es
}

dria contain their own DNA (mtDNA) and their own machinery for RNA and protein synthesis. The mitochondrial genome consists of 37 intronless genes, and encodes 13 subunits of the electron-transfer chain, 2 ribosomal RNAs (rRNA), and 22 transfer RNAs (tRNA). However, most of the mitochondrial proteins are encoded by genes in the nucleus and imported from the cytoplasm. Expression of the mitochondrial genome is 
initiated in the D-loop regulatory region of the mtDNA, and the basic transcription machinery consists of a mitochondrial RNA (mtRNA) polymerase molecule, a mt transcription factor A (mtTFA), and two related transcription factors B1 and B2 (mtTFB1 and mtTFB2) [13, 15,37]. All these mitochondrial transcription factors are encoded by nuclear genes, TFAM, TFB1M and TFB2M. The mtTFBs interact with the C-terminal tail of mtTFA, facilitating the interaction between the heavy and light-strand promoters and the initiation of the transcription [6,11]. In addition to its activity as a transcription factor, mtTFB1 belongs to the family of RNA adenine methyltransferases, a group of proteins that methylate small subunit rRNAs at a conserved stem loop [6].

Mutations in mtDNA are frequently linked to multisystemic diseases and follow a maternal transmission (mitochondria are inherited from the mother), while mutations in the nuclear genes encoding mitochondrial proteins cause typical mendelian traits $[2,10]$. These mtDNA mutations have an effect on cellular energy metabolism, and more severely affect those organs that are highly energy dependent, such as muscles and the nervous system. Cardiac muscle contraction is highly dependent on the energy supplied by mitochondria. The accumulation of mtDNA deletions is responsible for the age-related decrease in cardiac function, and some mtDNA mutations have been linked to hypertrophic cardiomyopathy (HCM), either isolated or as part of a multisystemic syndrome [19,21,33,40,41,44]. In addition to rare highly penetrant mutations, mtDNA is highly polymorphic and some single nucleotide polymorphisms (SNPs) could contribute to the expression of mitochondrial-related diseases [7,20].

Left ventricular hypertrophy (LVH) is caused by several factors that impose overwork to the heart, such as hypertension, valvular disease, cardiomyopathy, and myocardial infarction [26,29,36]. Hypertrophic cardiomyopathy (HCM) is the Mendelian autosomal dominant form of $\mathrm{LVH}$, and is primarily a disorder of the cardiac sarcomere linked to mutations in genes that encode components of the sarcomere, although other nonsarcomere genes could be involved in this disease [3, $4,30,38]$. Thus, polymorphisms in the genes encoding components of the renin-angiotensin and the endothelin systems, among others, could be involved in the risk of developing cardiac hypertrophy [5,8]. Further, some authors have reported the association between mtDNA variation and HCM $[2,7,33]$.

Every cell contains hundreds of mitochondria, and each mitochondriom about five mtDNA molecules.
The number of copies of mtDNA is directly dependent on the levels of mtTFA [23,24]. Mice heterozygous for a TFAM deletion showed a significant reduction in mtDNA copies [28]. The overexpression of mtTFA increased the number of copies, but did not affect respiratory chain activity or mitochondrial mass [11]. Mice with a deletion of TFAM limited to the heart reproduced the pathophysiological features of mitochondrial cardiomyopathy, and showed a significant reduction of mtDNA copies in cardiomyocytes with a partial reduction in respitarory chain activity $[27,42,43]$. The overexpression of mtTFB2 is accompanied by an increased amount of mtDNA, while cells that overexpressed mtTFB1 showed significantly increased mitochondrial mass $[9,12,31,43]$.

TFAM, TFB1M and TFB2M are thus candidate to be involved in the risk for developing cardiac hypertrophy. Therefore, we searched for DNA variation in these transcription factors in a cohort of well characterized HCM patients, and analysed the association of putative mutations with the disease through a case-control study.

\section{Methods}

\subsection{Patients and controls}

This study was part of a research to analyse the contribution of DNA variation to HCM. A total of 150 patients with HCM were recruited at the Cardiology Department of Hospital Central Asturias. The inclusion criteria were a left ventricular wall thickness $>$ $13 \mathrm{~mm}$ of unexplained cause: the hypertrophy was not secondary to other cardiac diseases capable of producing LVH, such as hypertension, valvular disease, and myocardial infarction. These patients were negative for mutations in the most frequently mutated sarcomeric genes (MYH7, TNNT2, MYBPC3, TNNI2, and TPM1). The main clinical and anthropometric characteristics of the 150 patients are summarised in Table 1. We also searched for TFAM, TFB1M, and TFB2M variants in a cohort of $50 \mathrm{HCM}$ patients from Germany with the same clinical and genetic inclusion criteria as the Spanish cohort.

A total of 250 healthy individuals, aged 20 to 75 years, were used as population controls for the genetic association studies. These controls were recruited through the blood bank and the Cardiology Dept. of Hospital Central Asturias and did not have a history of HCM. In 110 of these controls we performed elec- 
Table 1

Main characteristics of the 150 Spanish patients

\begin{tabular}{cc}
\hline Total & 150 \\
Male & $90(60 \%)$ \\
Age & $47 \pm 16(20-65)$ \\
Family history of HCM & $48(32 \%)$ \\
Mean body mass index & $27 \pm 5$ \\
\hline Mean LV septal thickness, mm & $19 \pm 6$ \\
range, mm & $13-42$ \\
First clinical symptom & \\
Dyspnea & $95(63 \%)$ \\
Arrhytmia & $23(15 \%)$ \\
Angina & $32(21 \%)$ \\
\hline
\end{tabular}

trocardiographic examination to exclude the existence of HCM or other cardiovascular diseases; in 140 controls, electro or echocardiographic studies were not performed to exclude the presence of asymptomatic LVH. All the Spanish patients and controls were Caucasians from the same region (Asturias, Northern Spain, total population 1 million). All the individuals participating in the study gave their informed consent, and the study was approved by the Ethical Committee of Hospital Central Asturias.

\subsection{Genetic analysis of TFAM, TFB1M and TFB2M}

Genomic DNA was obtained from $10 \mathrm{ml}$ of blood, and the coding regions of TFAM (7 exons), TFB1M (7 exons), and TFB2M (8 exons) were PCR-amplified in fragments 180 to 327 bp long. Each PCR consisted of $100 \mathrm{ng}$ of DNA, $2 \mathrm{mM} \mathrm{MgCl}{ }_{2}, 2 \mathrm{mM}$ of each dNTP, 10 pmol of each primer pair, and $0.5 \mathrm{U}$ of Taq DNApolymerase, in a final volume of $20 \mu \mathrm{l}$, and for a total of 33 PCR cycles $\left(30 \mathrm{~s}-95^{\circ} \mathrm{C}, 60 \mathrm{~s}\right.$-annealing temperature, $60 \mathrm{~s}-72^{\circ} \mathrm{C}$ ). Primer-sequences and PCR conditions are available upon request to the corresponding author. Nucleotide positions relative to the gene sequences were numbered according to the Ensembl database (www.ensembl.org). To search for sequence variants, the PCR fragments were amplified from all the patients and controls and analysed through single strand conformation analysis (SSCA) and denaturing high performance liquid chromatography (DHPLC).

For the SSCA, five $\mu$ l of each PCR were mixed with $30 \mu \mathrm{l}$ of formamide, denatured for $5 \mathrm{~min}$ at $95^{\circ} \mathrm{C}$, and $5 \mu \mathrm{l}$ were loaded on a $6 \%$ SSCA-polyacrylamide gel (5.8\% acrylamide/ $0.2 \%$ bisacrylamide; $50 \mathrm{~cm} \mathrm{long).}$ After $18 \mathrm{~h}$ of electrophoresis at $25 \mathrm{~W}$, gels were silverstained to visualise the electrophoretic patterns. For the DHPLC analysis, the PCR-products were injected in Helix DVB polymeric reversed-phase columns in a Varian Helix system, and eluted with a linear binary gradient created with buffers Varian Helix A (triethylammonium amine - TEAA) and B (TEAA $+25 \%$ acetonitrile) (www.varianinc.org). The DHPLC elution temperatures and buffers gradient for the PCR fragments were calculated with the DHPLC Melt Program (http://insertion.stanford.edu/melt.html), and are available upon request to the corresponding author.

To identify the nucleotide changes responsible for the observed genotypes, PCR fragments of cases representative for the different electrophoretic patterns were sequenced using BigDye chemistry in an automated ABI310 capillary system (Applied Biosystems, Foster City, CA, USA).

\subsection{Genotyping, haplotypes and linkage disequilibrium}

Because each allele gaves a characteristic SSCApattern, we could assign the genotype for each variant in all the patients and controls. Allele and genotype frequencies for the TFAM, TFB1M and TFB2M polymorphisms were determined in the Spanish patients and controls by SSCA-genotyping.

The frequencies for all the possible haplotypes defined by each pair of loci were calculated with the Cubic exact solutions for the estimation of pairwise haplotype frequencies (www.oege.org/software/cubex). This programm estimates the haplotype frequencies, the normalised linkage disequilibrium (LD) parameter (D'), and the LD correlation coefficient between two loci $\left(r^{2}\right)$ [17].

\subsection{Statistical analysis}

Allele and genotype frequencies in patients and controls were compared through a chi-square test. Odds ratios (OR) with $95 \%$ confidence intervals (CI) were obtained to calculate the relative risk of HCM associated with the genotypes. The $\mathrm{Chi}^{2}$ was also used to analyse the deviation from the Hardy-Weinberg equilibrium of the genotype frequencies. All statistical analyses were performed with the SPSS statistical package (v.11.0).

\section{Results}

We searched for TFAM, TFB1M and TFB2M variants in a total of $200 \mathrm{HCM}$ patients and 250 healthy controls. The coding exons and flanking intronic sequences were analysed through SSCA (Fig. 1), DHPLC (Fig. 2), and direct sequencing. 
Table 2

TFAM variants, and allele frequencies in our population. Nucleotides in exons were numbered considering +1 as the first base in codon 1 of the transcript (ENST00000373895). Nucleotides in introns were numbered according to the genomic sequence (ENSG00000108064).

\begin{tabular}{llcc}
\hline TFAM variants & Change & \multicolumn{2}{c}{ Allele frequencies } \\
\cline { 3 - 4 } & & Patients & Controls \\
\hline New, exon 1 & 5'UTR, -91 C $>$ A & $<1 \% *$ & 0 \\
rs1937, exon 1 & 35 C/G, S12T & C: 0.93 & 0.91 \\
& & G: 0.07 & 0.09 \\
rs17710934, exon 2 & 183 T/C, S61S & T: 0.95 & 0.93 \\
& & C: 0.05 & 0.07 \\
rs34084383, intron 3 & IVS3, -13 ins/del T & Tins: 0.20 & 0.22 \\
& & Tdel: 0.80 & 0.78 \\
new, exon 4 & 445 G $>$ A, A105T & $<1 \% *$ & 0 \\
rs41283688, intron 6 & IVS6, +12 G/A & A: 0.10 & 0.11 \\
& & G: 0.90 & 0.89 \\
\hline
\end{tabular}

*These variants were found in one patient and none of the 250 controls.

A
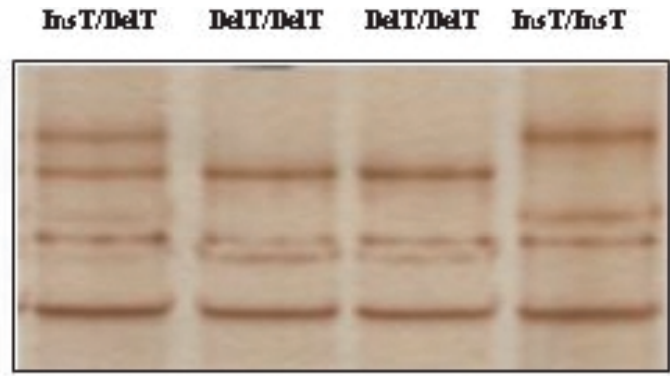

B

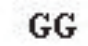

AG

AG

AA

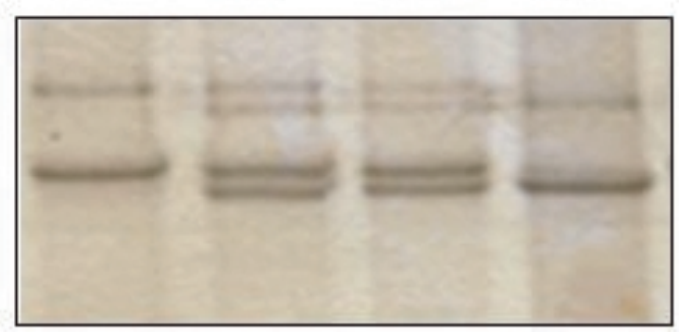

Fig. 1. (A) SSCA of polymorphism rs5782396 in TFB2M, showing the three genotypes for the IVS +26 ins/del T (intron 6). (B) SSCA of the exon 5 fragment, showing the different electrophoretic patterns for the $633 \mathrm{G} / \mathrm{A}$ polymorphism (rs324356).

\subsection{TFAM}

In the TFAM gene we identified four previously reported polymorphisms (Table 2). Among the two exon polymorphisms, rs17710934 was a silent change, and rs1937 was the only missense variant (S12T). Allele and genotype frequencies did not differ between patients and controls for the TFAM variants. We also determined the haplotype frequencies and linkage disequilibrium between these loci in the 150 patients and 250 controls from Spain. None of the TFAM polymor- phisms were in significant LD, and the stimated haplotype frequencies did not differ between patients and controls (Fig. 3).

In addition to the 4 common polymorphisms, we also found two rare TFAM variants in one patient and none of the healthy controls. The two changes were conserved among species. This patient was a 39 years old male with a left ventricular septum of $17 \mathrm{~mm}$ and was heterozygous for two nucleotide changes: -91 $\mathrm{C}>\mathrm{A}$ in the $5^{\prime}$-non translated (5'-UTR) sequence of exon 1, and a missense change in exon 4, A105T. This patient did not have symptoms of muscular or neurological disease. His mother was 82 years old and had a left ventricular septum (LVS) of $19 \mathrm{~mm}$. She was heterozygous for A105T, and in this way the patient should have the two rare variants in different chromosomes. No other family members were affected (the father died at the age of 60 without symptoms of HCM).

\subsection{TFB1M}

In the TFB1M gene we found five previously reported polymorphisms (Table 3 ). We also found two novel missense variants, a $\mathrm{G}>\mathrm{C}$ change in codon 120 (exon 3; A120 > P), and a G > A change in codon 189 (exon 5; G189 > R). These two changes were found in two patients and one control and therefore regarded as rare polymorphisms. Allele and genotype frequencies for the five common polymorphisms did not differ between patients and controls. The three exon 1 variants were in complete LD $\left(D^{\prime}=1.0 ; r^{2}=1.0\right)$, and in almost complete LD with rs324356. The stimated haplotype frequencies for each pair of loci did not differ between patients and controls (Fig. 3). 
Table 3

TFB1M polymorphisms, and allele frequencies in our population. $\mathrm{Nu}-$ cleotides in exons were numbered considering +1 as the first base in codon 1 of the transcript (ENST00000367166). Nucleotides in introns were numbered according to the genomic sequence (ENSG00000029639).

\begin{tabular}{llcc}
\hline TFB1M variants & Change & \multicolumn{2}{c}{ Allele frequencies } \\
\cline { 3 - 4 } & & Patients & Controls \\
\hline rs1334687, exon 1 & 5'UTR, -75 G/T & T: 0.46 & 0.58 \\
& & C: 0.56 & 0.42 \\
rs1334688, exon 1 & 5'UTR, -62 C/T & T: 0.44 & 0.48 \\
& & G: 0.58 & 0.58 \\
rs162981, exon 1 & 5'UTR, -23 G/T & T: 0.42 & 0.42 \\
& & C $<0.01$ & C $<0.01$ \\
New, exon 3 & 358 G/C, A120 $>$ P & A $<0.01$ & A $<0.01$ \\
New, exon 5 & 621 G $>$ A, G189 $>$ R & A: 0.42 & 0.42 \\
rs324356, exon 5 & 633 G/A, T211T & G: 0.58 & 0.58 \\
& & G $<1 \% *$ & 0 \\
New, exon 5 & 631 A $>$ G, T211 > A & A $<1 \% *$ & 0 \\
New, exon 6 & 767 G $>$ A, R256 $>$ Q & T: 0.41 & 0.42 \\
& & G: 0.59 & 0.58 \\
rs3734322, intron 6 & IVS6, -30 G/T &
\end{tabular}

$*$ these variants were found in one patient and none of the 250 controls.
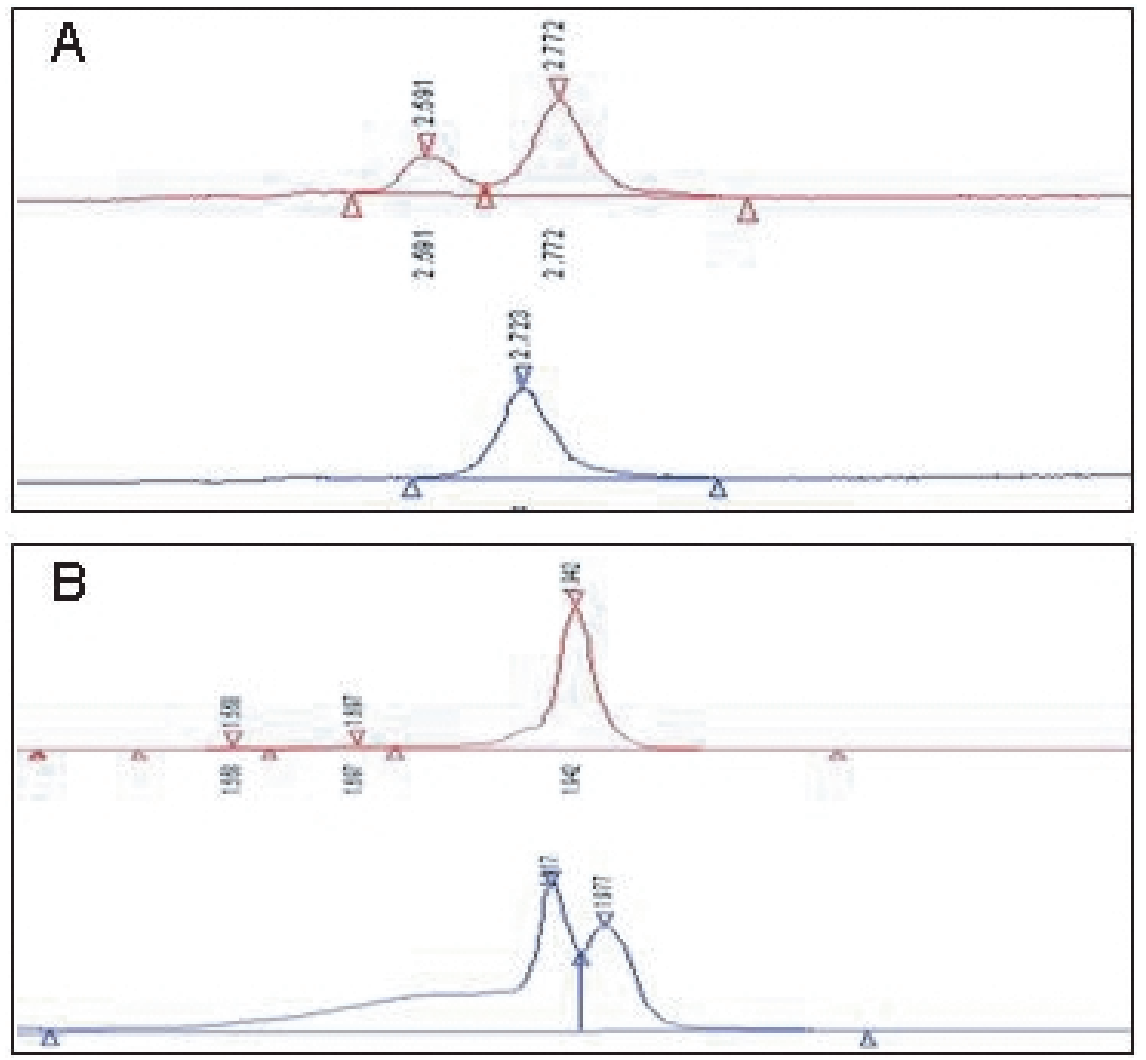

Fig. 2. DHPLC profile of the TFB1M exon 5 nucleotide variant (A631 > G), corresponding to the putative mutation Thr211 $>$ Ala. The PCR-amplified fragment of the heterozygous carrier eluted as two peaks, compared to the single peak in the wild-type homozygous.

Two sporadic cases had missense changes that were not found among the 250 controls. A 35 years old male with a LVS of $16 \mathrm{~mm}$ was heterozygous for a missense change in exon $5(631 \mathrm{~A}>\mathrm{G}$; T211 > A). A 25 years old female with a LVS of $15 \mathrm{~mm}$ was heterozygous for R256 $>\mathrm{Q}$ in exon $6(767 \mathrm{G}>\mathrm{A})$. These 

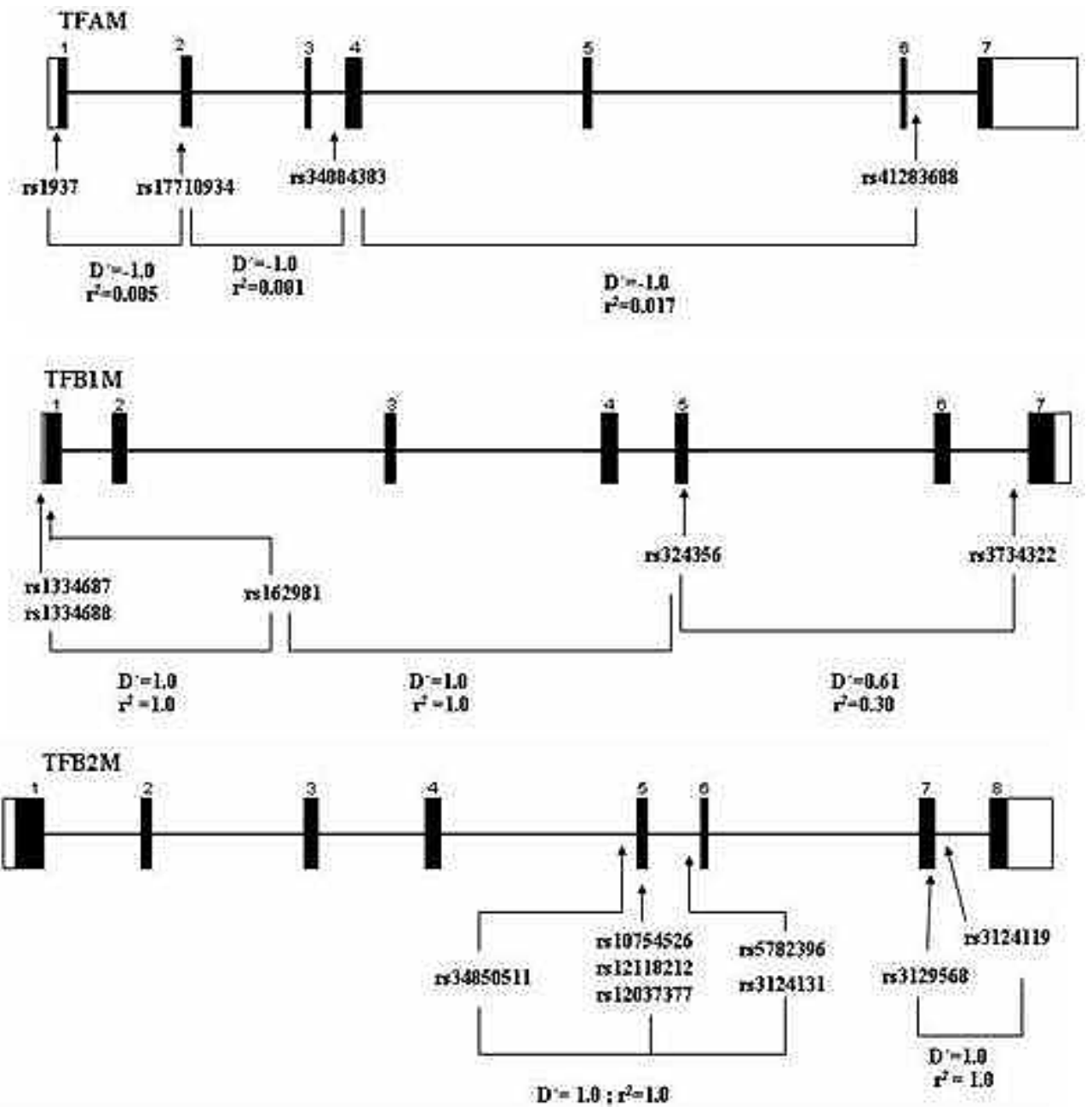

Fig. 3. Map of the TFAM, TFB1M and TFB2M showing the polymorphisms and the Linkage Disequilibrium values.

two TFB1M changes affected amino acids conserved among species.

\section{3. $T F B 2 M$}

In the TFB2M gene we found a total of 11 variants (Table 4). Three were novel polymorphisms: A64T (exon 1, $190 \mathrm{G} / \mathrm{A})$, IVS4 + $40 \mathrm{del} / \mathrm{ins}$ GT (intron4), and T392T (exon 8, $1176 \mathrm{C} / \mathrm{T}$ ). All the TFB2M variants were found in patients and controls, and A64 $>\mathrm{T}$ was a rare polymorphism found in two patients and one control. Allele, genotype, and haplotype frequencies for the TFB2M variants did not differ between patients and controls. LD analysis between pairs of loci showed three haplotype blocks: one defined by six polymorphisms between rs34850511 (intron 4) and rs3124131 (intron 6) and the other by rs3129568 and rs3124119 (intron 7) (Fig. 3).

\section{Discussion}

MtTFA facilitates the transcription of mtDNA, and is also important for the package of mtDNA in nucleoid particles [16,18,24,25,34]. Recent studies with transgenic mice demonstrated that mtTFA is important to maintain the number of mtDNA-copies, and its deletion was associated with a deficient respiratory chain activity and symptoms of dilated cardiomyopathy [42]. Moreover, this pathological phenotype was rescued by the expression of human mtTFA in the transgenic mice [22]. These findings suggested that mtTFA dysfunction could be involved in the development of cardiac hypertrophy in humans, and TFAM gene variants could be linked to the risk for HCM. Because mtTFA interacts with the mitochondrial transcription factors B1 and B2, and the cellular overexpression of these 
Table 4

TFB2M polymorphisms, and allele frequencies in our population. $\mathrm{Nu}$ cleotides in exons were numbered considering +1 as the first base in codon 1 of the transcript (ENST00000366514). Nucleotides in introns were numbered according to the genomic sequence (ENSG00000162851).

\begin{tabular}{llcc}
\hline TFB2M variants & Change & \multicolumn{2}{c}{ Allele frequencies } \\
\cline { 3 - 4 } & & Patients & Controls \\
\hline New, exon 1 & 190 G/A, A64T & A $<.01$ & $\mathrm{~A}<0.01$ \\
New, intron 4 & IVS4 +40 GT Ins/Del & ins: 0.98 & 0.95 \\
& & del: 0.02 & 0.05 \\
rs34850511, intron 4 & IVS4 -32 A/T & A: 0.57 & 0.55 \\
& & T: 0.43 & 0.45 \\
rs10754526, exon 5 & \multirow{2}{*}{ 723 C/T, P241P } & C: 0.57 & 0.55 \\
& & T: 0.43 & 0.45 \\
rs12118212, exon 5 & 783 A/T, K261K & A: 0.57 & 0.55 \\
& & T: 0.43 & 0.45 \\
rs12037377, exon 5 & 790 C/T, H264Y & C: 0.57 & 0.55 \\
& & T: 0.43 & 0.45 \\
rs5782396, intron 6 & IVS6 +26 ins/del T & insT:0.56 & 0.57 \\
rs3124131, intron 6 & IVS6 +58 T/A & delT: 0.44 & 0.43 \\
& & T: 0.56 & 0.57 \\
rs3129568, exon 7 & 984 T/C, F328F & A: 0.44 & 0.43 \\
& & C: 0.27 & 0.28 \\
rs3124119, intron 7 & IVS7 +7 A/G & T: 0.73 & 0.72 \\
& & G: 0.27 & 0.28 \\
New, exon 8 & 1176 C/T, T392T & A: 0.73 & 0.72 \\
& & T: 0.97 & 0.95 \\
& & & 0.03 \\
\hline
\end{tabular}

TFBs increases the amount of mtDNA and mitochondrial mass, mutations in TFB1M and TFB2M could also influence the risk for HCM [9,12,31].

We searched for TFAM, TFB1M and TFB2M gene variants in a cohort of HCM patients and controls. We found several common polymorphisms, with frequencies that did not differ between patients and controls, and thus would not contribute to the risk for cardiac hypertrophy. We also found four nucleotide changes in three patients, but none of the healthy controls. $\mathrm{Nu}-$ cleotide changes in candidate genes could be considered as putative mutations if they introduced amino acid changes that alter protein function, affect gene expression (promoter variation), or RNA-processing (intronic variants). In opposition to polymorphisms, mutations are rare among healthy controls, affect amino acids conserved among species, and segregate with the disease in affected families. Most of the patients in our study were sporadic cases, or from families with a limited number of patients (not big enough to perform a statistically significant linkage). In this way, the main reason to consider the two TFAM $(-91 \mathrm{C}>$ $\mathrm{A}$ and A105 $>\mathrm{T}$ ) and TFB1M (T211 > A and R256 $>$ Q) as putative mutations was their absence among the ethnically matched controls and the fact that they were highly conserved among species. The $-91 \mathrm{C}>$ $\mathrm{A}$ in TFAM was in a region that contains binding sites for transcription factors such as the nuclear respiratory factors. Amino acid 105 was in a high mobility group (HMG) domain of mtTFA. These HGM domains show high affinity binding for DNA sequences, and the Ala to Thr change could affect the binding of mtTFA to mtDNA $[11,35]$.

Human mtTFBs are closely related to RNA adenine methyltransferases, a group of proteins that methylate adenine residues at a conserved stem loop of rRNA molecules. This mtTFB dual-function as transcription activators and rRNA modifiers represents a potential link between transcription, ribosomal biogenesis, and translation [39]. There are no obvious reason why the two putative mutations in TFB1M could have a functional effect, but amino acid 211 is in a beta-sheet of the protein close to the seventh RNA methyltransferase motif. These changes could have effects on mtTFA and mtTFB1 expression/function, but functional studies are absolutelly necessary to confirm this point. In contrast with these rare variants found in the patients, the TFAM S12T alleles were frequent in patients and controls, and this amino acid was not conserved between species ( $\mathrm{S} / \mathrm{T}$ in human, $\mathrm{K}$ in mice). In addition, both variants would have similar mitochondrial signal peptide characteristics, hydropathy-scores, and average and local net charge [14].

In addition to TFB1M T211 > A and R256>Q, found in one patient and none of the controls, we also 
found three rare variants in two patients and one control: $\mathrm{A} 120>\mathrm{P}$ and $\mathrm{G} 189>\mathrm{R}$ in TFB1M, and $\mathrm{A} 64>\mathrm{T}$ in TFB2M. Amino acids 120 and 189 of mtTFB1 are highly conserved in mammals. However, their presence in one healthy control suggests that they could be rare variants not linked to HCM. The screening of more patients and controls should be necessary to define the association between these rare missense changes and HCM.

Finally, in addition to describe the variation at the TFAM, TFB1M and TFB2M in a Caucasion population, we also reported the linkage disequilibrium between the different polymorphisms at each gene. Haplotype frequencies and LD values did not differ between patients and controls, suggesting a non significant contribution to cardiac hypertrophy. However, the population genetics reported in our study could be usefull for future studies with diseases in which these mitochondrial transcription factors have been implicated, such as Alzheimer's [1].

Our work has several limitations. First, the main argument to consider the rare variants found in the patients as putative mutations, was their absence in the healthy controls. In addition, they affected amino acids conserved among species, suggesting a limitation for missense changes at these protein residues. However, functional studies are necessary to conclude that these variants affect the mitochondrial function. Second, the patients who had these rare variants were sporadic cases or from families with a reduced number of affected. This made impossible to stablish a familial segregation of the disease with these putative mutations. Third, the patients in our study had an essential form of left ventricular hypertrophy, and we can not exclude that the TFAM, TFB1M and TFB2M variants are involved in the risk for hypertrophy secondary to hypertension, cardiac valvular disease, exercise, or any other recognised cause of LVH. Fourth, SSCA and DHPLC are indirect techniques for detecting nucleotide changes in PCR-amplified fragments, and the existence of nucleotide changes that are not detected (approximatelly $5 \%$ of false negatives) is the main limitation for these methods. It is thus possible that some mutations/polymorphisms in the mitochondrial transcription factors were not identified in our study.

In conclusion, our work suggests that common DNA polymorphisms at the TFAM, TFB1M and TFB2M have no effect on risk for cardiac hypertrophy. We found rare variants in the TFAM and TFB1M that could contribute to the risk of developing HCM. However, the study of larger series of patients is necessary to clar- ify the role of mitochondrial transcription factors mutations in HCM. In addition, functional studies should be necessary to determine the biological effect of these rare putative mutations.

\section{Acknowledgements}

This work was supported by grants from the Spanish Fondo de Investigaciones Sanitarias-Fondos FEDER European Union (FIS-06/0214), and Red de Investigación Renal-REDINREN (RD06/0016).

\section{References}

[1] V. Alvarez, A.I. Corao, E. Sánchez-Ferrero, L. De Mena et al., Mitochondrial transcription factor A (TFAM) gene variation in Parkinson's disease, Neurosci Lett 423 (2008), 79-82.

[2] R. Anan, M. Nakagawa, M. Miyata, I. Higuchi, S. Nakao, M. Suehara, M. Osame and H. Tanaka, Cardiac involvement in mitochondrial diseases: a study of 17 patients with mitochondrial DNA defects, Circulation 91 (1995), 955-961.

[3] D.K. Arnett, L. de las Fuentes and U. Broeckel, Genes for left ventricular hypertrophy, Curr Hypertens Rep 6 (2004), 36-41.

[4] L.L. Bachinski and R. Roberts, Familial hypertrophic cardiomyopathy: diagnostic and therapeutic implications of recent genetic studies, Mol Med Today 2 (1996), 387-393.

[5] G.S. Bleumink, A.F.C. Schut, M.C.J.M. Sturkenboom, J.W. Deckers, C.M. van Duijn and B.H.C. Stricker, Genetic polymorphisms and heart failure, Genet Med 6 (2004), 465-474.

[6] N.D. Bonawitz, D.A. Clayton and G.S. Shadel, Initiation and beyond: multiple functions of the human mitochondrial transcription machinery, Mol Cell 24 (2006), 813-825.

[7] M.G. Castro, C. Huerta, J.R. Reguero, M.I. Soto, E. Domenech, V. Alvarez, M. Gomez-Zaera, V. Nunes, P. Gonzalez, A. Corao and E. Coto, Mitochondrial DNA haplogroups in Spanish patients with hypertrophic cardiomyopathy, Int $J$ Cardiol 112 (2006), 202-206.

[8] M.G. Castro, F. Rodriguez-Pascual, N. Magan-Marchal, J.R. Reguero, C. Alonso-Montes, C. Moris, V. Alvarez, S. Lamas and E. Coto, Screening of the endothelin1 gene (EDN1) in a cohort of patients with essential left ventricular hypertrophy, Ann Hum Genet 71 (2007), 601-610.

[9] J. Cotney, Z. Wang and G.S. Shadel, Relative abundance of the human mitochondrial transcription system and distinct roles for h-mtTFB1 and h-mtTFB2 in mitochondrial biogenesis and gene expression, Nucleic Acids Res 18 (2007), 1-13.

[10] S. DiMaur and E. Schon, Mitochondrial respiratory-chain disases, $N$ Engl J Med 348 (2003), 2656-2668.

[11] M.I. Ekstrand, M. Falkenberg, A. Rantanen, C.B. Park, M. Gaspari, K. Hultenby, P. Rustin, C.M. Gustafsson and N.G. Larsson, Mitochondrial transcription factor A regulates $\mathrm{mtD}$ NA copy number in mammals, Hum Mol Genet 13 (2004), 935-944.

[12] M. Falkenberg, M. Gaspari, A. Rantanen, A. Trifunovic, N.G. Larsson and C.M. Gustafsson, Mitochondrial transcription factors B1 and B2 activate transcription of human mtDNA, Nat Genet (2002), 289-294. 
[13] R.P. Fisher and D.A. Clayton, Purification and characterization of human mitochondrial transcription factor 1, Mol Cell Biol 8 (1988), 3496-3509.

[14] C. Günther, K. von Hadeln, T. Müller-Thomsen, A. Alberici, G. Binetti and C. Hock et al., Possible association of mitochondrial transcription factor A (TFAM) genotype with sporadic Alzheimer disease, Neurosci Lett 369 (2004), 219-223.

[15] H.L. Garstka, W.E. Schmitt, J. Schultz, B. Sogl et al., Import of mitochondrial transcription factor A (TFAM) into rat liver mitochondria stimulates transcription of mitochondrial DNA, Nucleic Acids Res 31 (2003), 5039-5047.

[16] M. Gaspari, M. Falkenberg, N.G. Larsson and C.M. Gustafsson, The mitochondrial RNA polymerase contributes critically to promoter specificity in mammalian cells, EMBO $J \mathbf{2 3}$ (2004), 4606-4614.

[17] T.R. Gaunt, S. Rodriguez and I.N.M. Day, Cubic exact solutions for the estimation of pairwise haplotype frequencies: implications for linkage disequilibrium analyses and a web tool 'CubeX', BMC Bioinformatics 8 (2007), 428.

[18] S.C. Ghivizzani, C.S. Madsen, M.R. Nelen, C.V. Ammini and W.W. Hauswirth, In organello footprint analysis of human mitochondrial DNA: human mitochondrial transcription factor A interactions at the origin of replication, Mol Cell Biol 14 (1994), 7717-7730.

[19] K. Hattori, M. Tanaka, S. Sugiyama, T. Obayashi, T. Ito, T. Satake et al., Age-dependent increase in deleted mitochondrial DNA in the human heart: possible contributory factor in presbycardia, Am Heart J 121 (1991), 1735-1742.

[20] S. Hofmann, M. Jaksch, R. Bezold, S. Mertens, S. Aholt, A. Paprotta and K.D. Gerbitz, Population genetics and disease susceptibility: characterization of central European haplogroups by mtDNA gene mutations, correlation with D loop variants and association with disease, Hum Mol Genet 6 (1997), 1835-1846.

[21] R.H. Hsieh, J.Y. Li, C.Y. Pang and Y.H. Wei, A novel mutation in the mitochondrial 16S rRNA gene in a patient with MELAS syndrome, diabetes mellitus, hyperthyroidism and cardiomyopathy, J Biomed Sci 8 (2002), 328-335.

[22] M. Ikeuchi, H. Matsusaka, D. Kang, S. Matsushima, T. Ide, T. Kubota et al., Overexpression of mitochondrial transcription factor a ameliorates mitochondrial deficiencies and cardiac failure after myocardial infarction, Circulation 112 (2005), 683-690.

[23] D. Kang and N. Hamasaki, Mitochondrial transcription factor A in the maintenance of mitochondrial DNA, Ann NY Acad Sci 1042 (2005), 101-108.

[24] D. Kang, S.H. Kim and N. Hamasaki, Mitochondrial transcription factor A (TFAM): roles in maintenance of mtDNA and cellular functions, Mitochondrion 7 (2007), 39-44.

[25] BA. Kaufman, N. Durisic, J.M. Mativetsky, S. Costantino, M.A. Hancock, P. Grutter and E.A. Shoubridge, The mitochondrial transcription factor TFAM coordinates the assembly of multiple DNA molecules into nucleoid-like structures, $\mathrm{Mol}$ Biol Cell 18 (2007), 3225-3236.

[26] I. Komuro and Y. Yazaki, Control of cardiac gene expression by mechanical stress, Annu Rev Physiol (1993), 55-75.

[27] N.G. Larsson, J. Wang, H. Wilhelmsson, A. Oldfors, P. Rustin, M. Lewandoski, G.S. Barsh and D.A. Clayton, Mitochondrial transcription factor A is necessary for mtDNA maintenance and embryogenesis in mice, Nat Genet 18 (1998), 231-236.

[28] H. Li, J. Wang, H. Wilhelmsson, A. Hansson, P. Thoren, J. Duffy, P. Rustin and N.G. Larsson, Genetic modification of survival in tissue-specific knockout mice with mitochondrial cardiomyopathy, Proc Natl Acad Sci USA 97 (2000), 34673472 .

[29] B.H. Lorell and B.A. Carabello, Left ventricular hypertrophy: pathogenesis, detection, and prognosis, Circulation 102 (2000), 470-479.

[30] A.J. Marian and R. Roberts, Recent advances in the molecular genetics of hypertrophic cardiomyopathy, Circulation $\mathbf{9 2}$ (1995), 1336-1347.

[31] V. McCulloch, B.L. Seidel-Rogol and G.S. Shadel, A human mitochondrial transcription factor is related to RNA adenine methyltransferases and binds S-adenosylmethionine, Mol Cell Biol 22 (2002), 1116-1125.

[32] V. McCulloch and G.S. Shadel, Human mitochondrial transcription factor B1 interacts with the C-terminal activation region of h-mtTFA and stimulates transcription independently of its RNA methyltransferasa activity, Mol Cell Biol 23 (2003), 5816-5824.

[33] T. Obayashi, K. Hattori, S. Sugiyama, M. Tanaka, T. Tanaka, S. Itoyama et al., Point mutations in mitochondrial DNA in patients with hypertrophic cardiomyopathy, Am Heart J 124 (1992), 1263-1269.

[34] K. Ohgaki, T. Kanki, A. Fukuoh, H. Kurisaki, Y. Aoki, M. Ikeuchi et al., The C-terminal tail of mitochondrial transcription factor A markedly strengthens its general binding to DNA, J Biochem 141 (2007), 201-211.

[35] C.A. Piantadosi and H.B. Suliman, Mitochondrial transcription factor A induction by redox activation of nuclear respiratory factor 1, J Biol Chem 281 (2006), 324-333.

[36] J. Sadoshima and S. Izumo, The cellular and molecular response of cardiac myocytes to mechanical stress, Annu Rev Physiol 59 (1997), 551-571.

[37] R.C. Scarpulla, Nuclear activators and coactivators in mammalian mitochondrial biogenesis, Biochim Biophys Acta 1576 (2002), 1-14.

[38] K. Schwartz, L. Carrier, P. Guicheney and M. Komajda, Molecular basis of familial cardiomyopathies, Circulation 91 (1995), 532-540.

[39] B.L. Seidel-Rogol, V. McCulloch and G.S. Shadel, Human mitocondrial transcription factor B1 methylates ribosomal RNA at a conserved stem loop, Nat Genet 33 (2003), 23-24.

[40] G. Silvestri, F.M. Santorelli, S. Shanske, C.B. Whitley, L.A. Schimmenti, S.A. Smith and S. DiMauro, A new DNA mutation in the tRNA leu (UUR) gene associated with maternally inherited cardiomyopathy, Hum Mutat 3 (1994), 37-43.

[41] S. Sugiyama, K. Hattori, M. Hayakawa, T. Ozawa, Quantitative analysis of age-associated accumulation of mitochondrial DNA with deletion in human hearts, Biochem Biophys Res Commun 180 (1991), 894-899.

[42] J. Wang, H. Wilhelmsson, C. Graff, H. Li, A. Oldfors, P. Rustin et al., Dilated cardiomyopathy and atrioventricular conduction blocks induced by heart-specific inactivation of mitochondrial DNA gene expression, Nat Genet 21 (1999), 133-137.

[43] A. Wredenberg, R. Wibom, H. Wilhelmsson, C. Graff, H.H. Wiener, S.J. Burden et al., Increased mitochondrial mass in mitochondrial myopathy mice, Proc Natl Acad Sci USA 99 (2002), 15066-15071.

[44] M. Zeviani, C. Gellera, C. Antozzi, M. Rimoldi, L. Morandi, F. Villani, V. Tiranti and S. DiDonato, Maternally inherited myopathy and cardiomyopathy: association with mutation in mitochondrial DNA tRNA leu, Lancet 338 (1991), 143-147. 


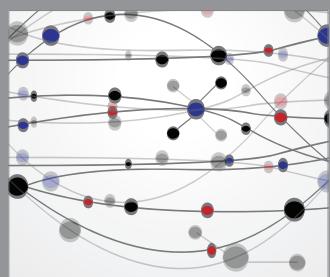

The Scientific World Journal
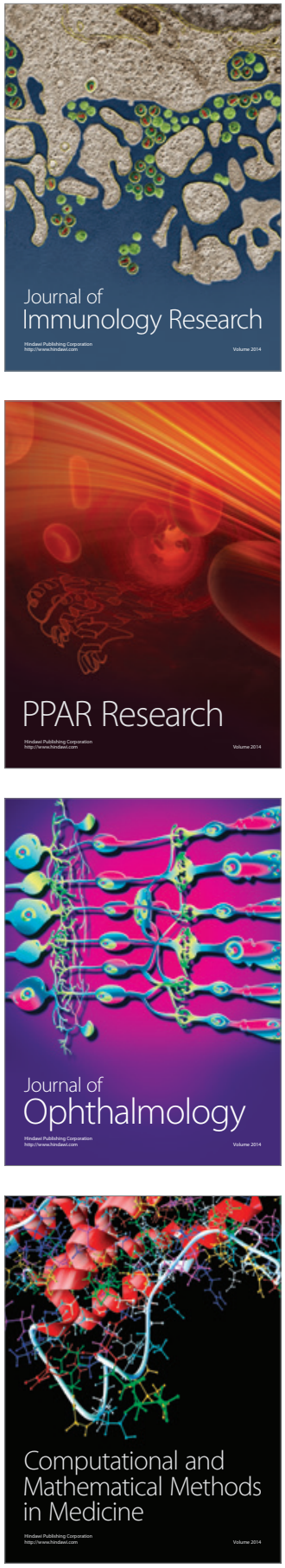

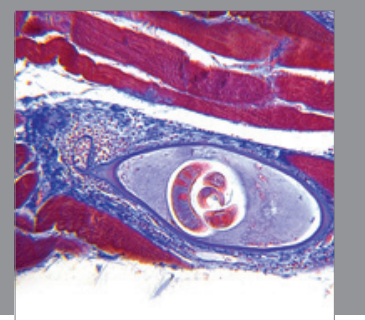

Gastroenterology

Research and Practice
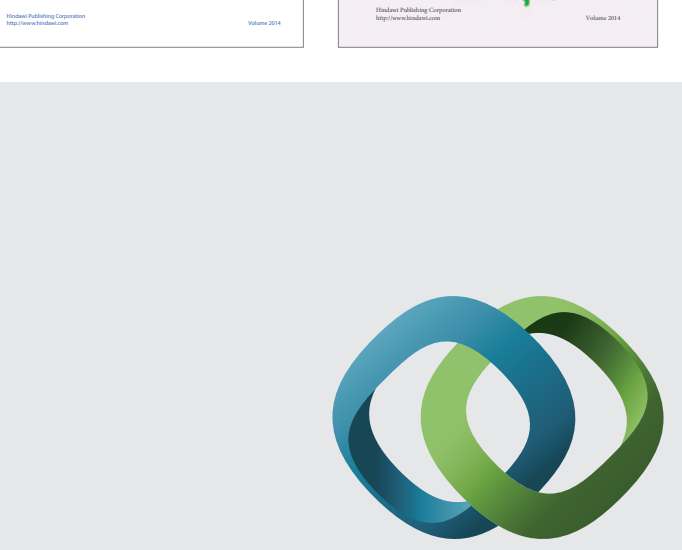

\section{Hindawi}

Submit your manuscripts at

http://www.hindawi.com
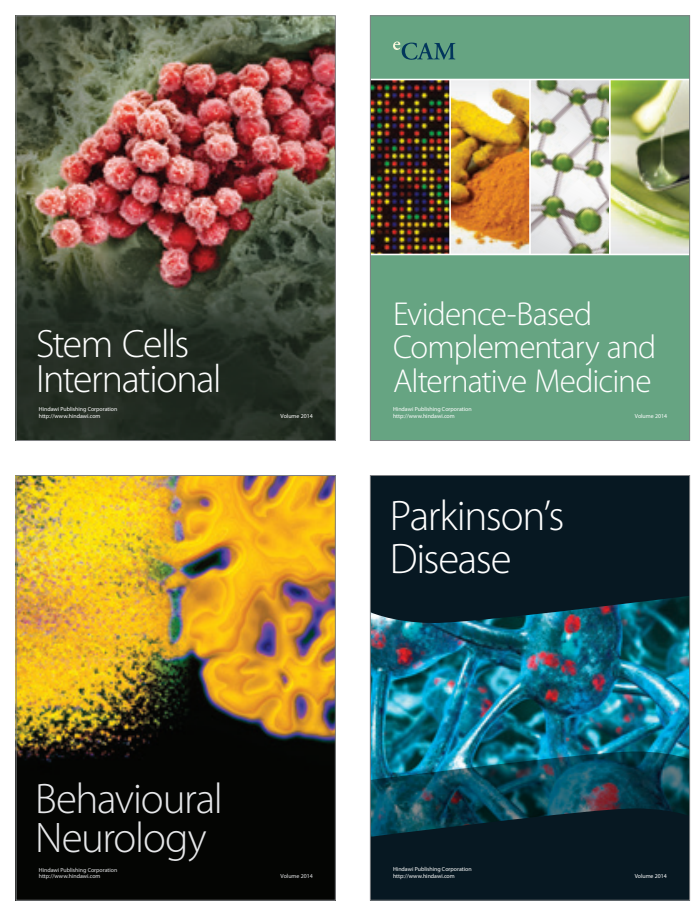

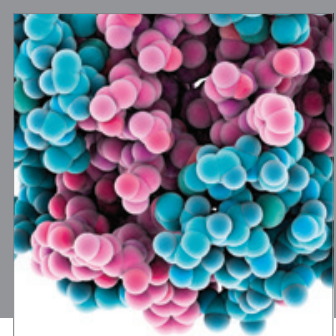

Journal of
Diabetes Research

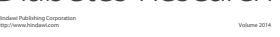

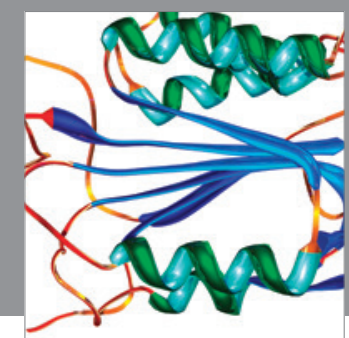

Disease Markers
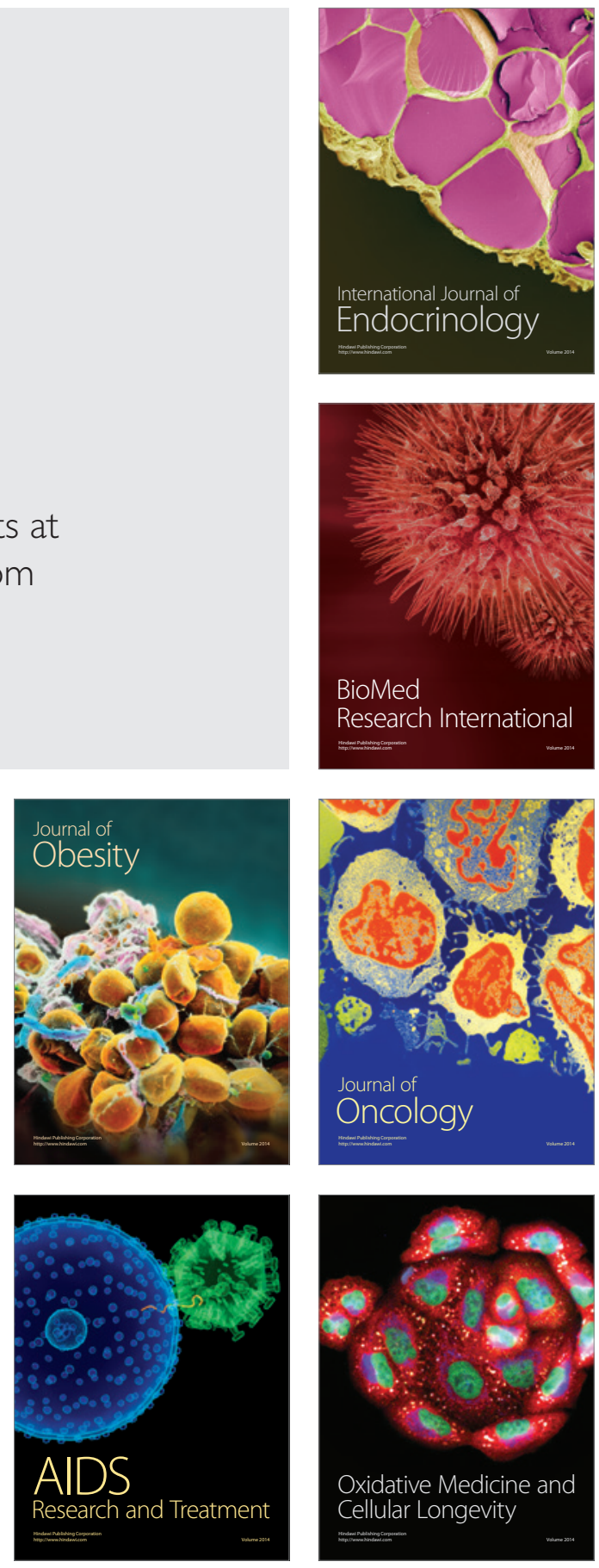